\title{
The electron impact broadening parameters in hot star atmospheres: Mn II, Mn III, Ga III, Ge III and Ge IV lines ${ }^{\star}$
}

\author{
L.Č. Popović and M.S. Dimitrijević \\ Astronomical Observatory, Volgina 7, 11050 Beograd, Serbia, Yugoslavia
}

Received June 5; accepted June 6, 1997

\begin{abstract}
The electron - impact line widths and shifts for 16 Mn II, 3 Mn III, 10 Ga III, 8 Ge III and 14 Ge IV multiplets, have been considered within a modified semiempirical approach. Moreover, using a semiclassical approach, we have considered electron-, proton-, and He III-impact line widths and shifts for $3 \mathrm{Ge}$ IV multiplets. The obtained results have been compared with other theoretical results.
\end{abstract}

Key words: lines: profile-atomic data — stars: chemically peculiar

\section{Introduction}

Lines of doubly- and triply-charged emitters are present in atmospheres of hot (A and B) stars $\left(T_{\text {eff }} \gtrsim 10000 \mathrm{~K}\right)$ where Stark broadening mechanism is important. Moreover for such plasma conditions, it is the main pressure broadening mechanism. Also, as it was shown in Popović \& Dimitrijević (1996a), in stellar atmospheres there exist conditions where Stark widths are comparable and even up to one order magnitude larger than the corresponding thermal Doppler widths. Consequently, Stark width data for a large number of transitions in many atomic and ionic spectra are needed for modeling of hot stellar plasma. Even in cooler stars, Stark broadening data for multicharged ion species may be of interest for the modeling of subphotospheric layers, for radiative transfer and opacity calculations, as well as for further developement of the physics of stellar interiors (Seaton 1987).

The increase of the number of spectral lines of astrophysical interest is additionally stimulated with the development of the space observations. Leckrone et al.

Send offprint requests to: L.Č. Popović

* Tables 1-4 are only available in electronic form via anonymous ftp 130.79.128.5 at the CDS in Strasbourg or via http:// cdsweb.u-strasbg.fr/Abstract.html
(1993) investigating chemically peculiar stars with the Goddard High Resolution Spectrograph (GHRS) on the Hubble Space Telescope (HST) have found that "begining at $Z=32$ (germanium) and extending to heavier elements, there is a dramatic increase in the magnitude of overabundance. All species between $Z=32$ and $Z=56$ analysed are overabundant relative to the Sun".

Here we present Stark broadening parameters for three ionized elements which lines are present in spectra of hot star atmospheres. Manganese is overabundant in atmospheres of Hg-Mn stars (Heacox 1979; Cowley 1980; Smith \& Dworetsky 1993 etc.), and lines of singly and doubly charged manganese ions are observed in stellar atmospheres (Heacox 1979; Cowley 1980; Smith \& Dworetsky 1993). Takada-Hidai et al. (1986) have analysed Ga II and Ga III lines in high resolved IUE spectra observed for 53 $\mathrm{B}$ and A stars and they have found an overabundance of gallium in the magnetic Si and Si Cr Eu stars, in the nonmagnetic Hg-Mn stars and in the He-weak PGa stars (for gallium overabundance analyses see also e.g. Heacox 1979 and Smith 1995). They have used for the spectrum synthesis models with $T_{\text {eff }}=11000 \mathrm{~K}-17000 \mathrm{~K}$ and $\log g=3.5$, where the Stark broadening is the main pressure broadening mechanism and where the inclusion of this mechanism is of importance (Dimitrijević \& Artru 1986). Moreover, Smith (1995) underlines the importance of gallium spectral lines for the detection of stratification effects in HgMn stars.

Spectral lines of germanium (Ge II, Ge III) are present in hot star spectra, as e.g. in $\beta$ Ori (Selvelli et al. 1977) spectrum. They may be additionally of interest for stellar interior physics since germanium is commonly associated with slow-neutron capture nucleosynthesis (Leckrone et al. 1993).

In order to provide to astrophysicists the Stark broadening data needed for stellar spectra analysis and synthesis as well as for astrophysical and physical plasmas research and modelling, an effort has been made (see Dimitrijević 1996 and references therein) to obtain the 
$4 s-4 p(120.23 \mathrm{~nm})$

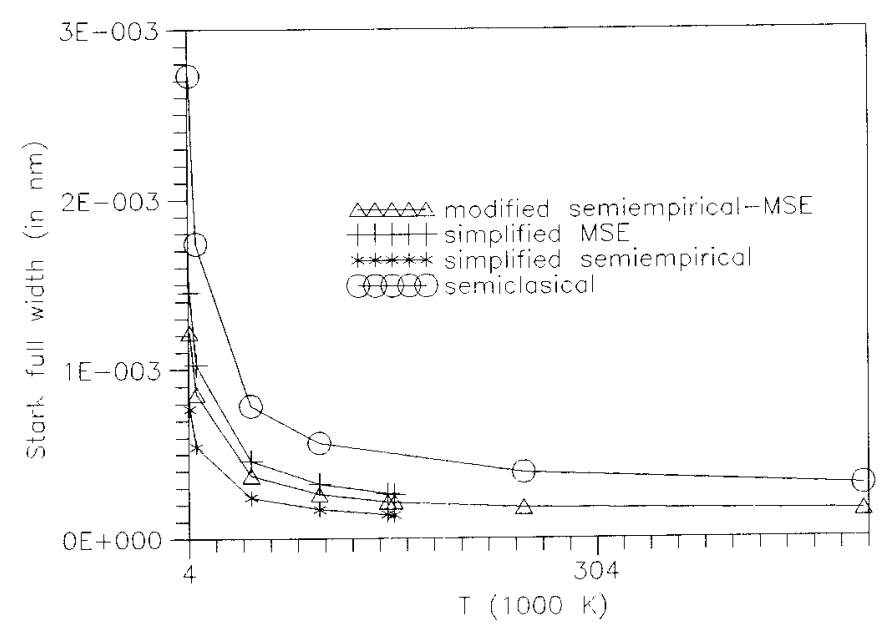

Fig. 1. Stark full widths for $4 \mathrm{~s}^{2} \mathrm{~S}-4 \mathrm{p}^{2} \mathrm{P}^{0}$ transition of Ge IV calculated by using different approximations, as functions of temperature. The electron density is $10^{23} \mathrm{~m}^{-3}$

needed data within the semiclassical perturbation approach, developed by Sahal-Bréchot (1969a,b) and improved and updated several times (Sahal-Bréchot 1974; Fleurier et al. 1977; Dimitrijević \& Sahal-Bréchot 1984 1995; Dimitrijević et al. 1991). In the case where the semiclassical perturbation approach is not applicable with an appropriate accuracy due to the lack of reliable atomic energy levels data, the simpler, modified semiempirical approach (Dimitrijević \& Konjević 1980) has been applied.

Table 5. Stark widths for Mn II $a^{5} \mathrm{D}-\mathrm{z}^{5} \mathrm{P}^{0}$ multiplet obtained by using different approximations (Dimitrijević 1982). Used notation: $W_{\mathrm{MSE}}$ - calculation with the modified semiempirical approach (Dimitrijević \& Konjević 1980), $W_{\mathrm{SE}}-$ semiempirical approach (Griem 1968), $W_{\mathrm{SC}}$ - semiclassical theory of Jones et al. (1971)

\begin{tabular}{lllll}
\hline Transition & $T(\mathrm{~K})$ & $W_{\mathrm{SEM}}(\mathrm{nm})$ & $W_{\mathrm{SE}}(\mathrm{nm})$ & $W_{\mathrm{SC}}(\mathrm{nm})$ \\
\hline $\mathrm{a}^{5} \mathrm{D}-\mathrm{z}^{5} \mathrm{P}^{0}$ & 2500 & .0246 & .0166 & .0266 \\
$\mathrm{Mn} \mathrm{II}$ & 5000 & .0174 & .0117 & .0190 \\
$\bar{\lambda}=346.4 \mathrm{~nm}$ & 10000 & .0123 & .0083 & .0144 \\
& 20000 & .00870 & .00587 & .0114 \\
\hline
\end{tabular}

Here we have calculated within the semiclassical perturbation formalism (Sahal-Bréchot 1969a,b) electron-, proton-, and He III-impact line widths and shifts for 3 Ge IV lines. Moreover, Stark widths and shifts for $16 \mathrm{Mn}$ II, 3 Mn III, 7 Ga III, 8 Ge III and 13 Ge IV lines for which there is not a set of reliable atomic energy level data sufficiently complete to achieve the appropriate accuracy of the semiclassical perturbation method, have been calculated
Table 6. Same as in Table 5 , but for Ga III $4 \mathrm{~s}^{2} \mathrm{~S}-4 \mathrm{p}^{2} \mathrm{P}^{0}$ multiplet (Dimitrijević \& Artru 1986). $W_{\mathrm{G}}$ - aproximate semiclassical method (Griem 1974)

\begin{tabular}{lllll}
\hline Transition & $T(\mathrm{~K})$ & $W_{\mathrm{SEM}}(\mathrm{nm})$ & $W_{\mathrm{SE}}(\mathrm{nm})$ & $W_{G}(\mathrm{~nm})$ \\
\hline $4 \mathrm{~s}^{2} \mathrm{~S}-4 \mathrm{p}^{2} \mathrm{P}^{0}$ & 2500 & .00325 & .00229 & .00436 \\
Ga III & 5000 & .00230 & .00162 & .00311 \\
$\bar{\lambda}=150.8 \mathrm{~nm}$ & 10000 & .00163 & .00114 & .00223 \\
& 20000 & .00115 & .000808 & .00163 \\
& 40000 & .000814 & .000571 & .00123 \\
\hline
\end{tabular}

within the modified semiempirical approach (Dimitrijević \& Konjević 1980 and for ions with complex spectra see also Popović \& Dimitrijević 1996b).

\section{Results and discussion}

Summaries of the semiclassical perturbation formalism (Sahal-Bréchot 1969a,b) and of the modified semiempirical formalism (Dimitrijević \& Konjević 1980) are given in Dimitrijević \& Sahal-Bréchot (1995) and Popović \& Dimitrijević (1996b) and will not be repeated here. The atomic energy levels needed for calculations were taken from Bashkin \& Stoner (1987) for Mn II, Moore (1971) for Mn III, Ryabtsev \& Wyart (1987) and Isberg \& Litzén (1986) for Ga III, Sugar \& Musgrove (1993) for Ge III, and in Ryabtsev \& Wyart (1987) and Sugar \& Musgrove (1993) for Ge IV. Oscillator strengths have been calculated by using the method of Bates \& Damgaard (1949) and the tables of Oertel \& Shomo (1968). For higher levels, the method described by Van Regemorter et al. (1979) has been used.

Results obtained within the modified semiempirical approach - MSE (Dimitrijević \& Konjević 1980) for electron - impact line widths and shifts for $16 \mathrm{Mn} \mathrm{II,} 3 \mathrm{Mn}$ III, $10 \mathrm{Ga}$ III, $8 \mathrm{Ge}$ III and $14 \mathrm{Ge}$ IV multiplets as a function of temperature are presented in Tables $1-3$ (accessibles only in electronic form). The calculations were performed for the perturber density of $10^{23} \mathrm{~m}^{-3}$. Within the used approach, results for electron-impact broadening parameters are linear with electron density. For three lines of Ge IV it is possible to calculate Stark broadening parameters within the semiclassical perturbation approach (Sahal-Bréchot 1969a,b). For electron-, proton-, and He III-impact line widths and shifts for $3 \mathrm{Ge}$ IV multiplets for the perturber densities of $10^{23}-10^{26} \mathrm{~m}^{-3}$ and temperatures $T=5000-500000 \mathrm{~K}$, are shown in Table 4 (accessible only in electronic form). For electron densities lower than tabulated, a linear interpolation with density is sufficient. We also specify a parameter $C$ (Dimitrijević \& Sahal-Bréchot 1984), which gives an estimate for the maximum perturber density for which the line may be treated as isolated when it is divided by the corresponding full width at half maximum. For each value given 
in Table 1, the collision volume $(V)$ multiplied by the perturber density $(N)$ is much less than one and the impact approximation is valid (Sahal-Bréchot 1969a,b). Values for $N V>0.5$ are not given and values for $0.1<$ $N V \leq 0.5$ are denoted by an asterisk. For higher densities, when the impact approximation is not valid, the ion broadening contribution may be estimated by using quasistatic approach (Sahal-Bréchot 1991 or Griem 1974). In the region between where neither of these two approximations is valid, a unified type theory should be used. For example in Barnard et al. (1974), simple analytical formulas for such a case are given. The accuracy of the results decreases when broadening by ion interactions becomes important. As an illustration, in Fig. 1. we have compared Stark widths calculated by using the semiclassical, MSE, simplified MSE and Griem's semiempirical appraoch.

In the case of these ions there is not measured Stark broadening parameters. For Mn II $\mathrm{a}^{5} \mathrm{D}-\mathrm{z}^{5} \mathrm{P}^{0}$ transition Dimitrijević (1982) performed calculations by using the MSE, Griem's semiempirical approach - SE (Griem 1968) and semiclassical approach of Griem (see Jones et al. 1971; Griem 1974). Since these data complete MSE results for Mn II they are shown here in Table 5, together with semiempirical and semiclassical results. The MSE results are in good agreement with more sophisticated semiclassical calculations. For Ga III $4 \mathrm{~s}^{2} \mathrm{~S}-4 \mathrm{p}^{2} \mathrm{P}^{0}$ multiplet calculations by using the MSE and the SE approach as well as calculations by using approximate semiclassical method (Griem 1974) and the modification of this method (Dimitrijević \& Konjević 1980) were performed by Dimitrijević \& Artru (1986). For this multiplet calculations within the SE approach are given in Puric et al. (1978) as well. Results according to the approximate semiclassical method are slightly larger than MSE results and results obtained by the modification of approximate semiclassical method which are in good agreement.

Also, for Mn II $\mathrm{a}^{7} \mathrm{~S}-\mathrm{z}^{7} \mathrm{P}^{0}$ multiplet, width and shift estimated on the basis of Stark broadening parameters dependence on ionized potential from the lower level of the corresponding transition exist as well (Lakićević 1983). For an electron density of $10^{23} \mathrm{~m}^{-3}$ and an electron temperature of $20000 \mathrm{~K}$, Lakićević obtains $W_{\mathrm{L}}=0.008 \mathrm{~nm}$ and $|d|_{\mathrm{L}}=0.0036 \mathrm{~nm}$, while our results are $W_{\mathrm{MSE}}=$ $0.0041 \mathrm{~nm}$ and $|d|_{\mathrm{MSE}}=0.00093 \mathrm{~nm}$.

We hope that Stark broadening data presented here will be of interest for space spectroscopy and for chemically peculiar star research as well as for laboratory plasma spectroscopy. Experimental data for considered ion lines will be of interest for the discussion and developement of Stark broadening theory for heavier emitters with more complex spectra.

Acknowledgements. This work is a part of the project "Astrometrical, Astrodynamical and Astrophysical Investigations", supported by Ministry of Science and Technology of Serbia.

\section{References}

Barnard A.J., Cooper J., Smith E.W., 1974, J. Quant. Spectrosc. Radiat. Transfer 14, 1025

Bashkin S., Stoner J.O., 1987, Atomic energy levels and Grotrian diagrams 2. North-Holland Publishing Company, Amsterdam, Oxford, New York

Bates D.R., Damgaard A., 1949, Phil. Trans. Roy. Soc. London, Ser. A 242, 101

Cowley C.R., 1980, PASP 92, 159

Dimitrijević M.S., 1982, in: Sun and Planetary System, Fricke W. and Teleki G. (eds.). D. Reidel Publishing Company, p. 101

Dimitrijević M.S., 1996, Zh. Prikl. Spektrosk. 63, 810

Dimitrijević M.S., Artru M.-C., 1986, The Physics of Ionized Gases, Contributed papers of SPIG'86, Šibenik, Kurepa M.V. (ed.), Dept. of Physics and Meteorology, University of Belgrade, p. 317

Dimitrijević M.S., Konjević N., 1980, J. Quant. Spectrosc. Radiat. Transfer 24, 451

Dimitrijević M.S., Sahal-Bréchot S., 1984, J. Quant. Spectrosc. Radiat. Transfer 31, 301

Dimitrijević M.S., Sahal-Bréchot, S., 1995, Phys. Scr. 52, 41

Dimitrijević M.S., Sahal-Bréchot S., Bommier V., 1991, A\&AS 89,581

Griem H.R., 1968, Phys. Rev. 165, 258

Griem H.R., 1974, Spectral Line Broadening by Plasmas. Academic Press, New York

Fleurier C., Sahal-Bréchot S., Chapelle J., 1977, J. Quant. Spectrosc. Radiat. Transfer 17, 595

Heacox W.D., 1979, ApJS 41, 675

Isberg B., Litzén U., 1986, Phys. Scr. 33, 420

Jones W.W., Benett S.M., Griem H.R., Univ. of Meryland Techn. Rep. No 1971-128, College Park, Maryland

Lakićevć I.S., 1983, A\&A 127, 37

Leckrone D.S., Wahlgren G.M., Johansson S.G., Adelman S.J., 1993, in Peculiar versus Normal Phenomena in A-Type and Related Stars, Dworetsky M.M., Castelli F., Faraggiana R. (eds.), ASP Conf. Ser. 44, 42

Migdalek By.J., 1997, Acta Phys. Polonica A52, 465

Moore C.E., 1971, Atomic Energy Levels, NSRDS- NBS, Vol. 2, Washington D.C.

Oertel G.K., Shomo L.P., 1968, ApJS 16, 175

Popović L.Č., Dimitrijević M.S., 1996a, A\&AS 120, 373

Popović L. Č., Dimitrijević M.S., 1996b, Phys. Scr. 53, 325

Purić J., Dimitrijević M.S., Lakićević I.S., 1978, Phys. Lett. $67 \mathrm{~A}, 189$

Ryabtsev A.N., Wyart J.-F., 1987, Phys. Scr. 36, 255

Sahal-Bréchot S., 1969a, A\&A 1, 91

Sahal-Bréchot S., 1969b, A\&A 2, 322

Sahal-Bréchot S., 1974, A\&A 35, 321

Seaton M.J., 1987, J. Phys. B 20, 6363

Selvelli P.L., Crivellari L., Stalio R., 1977, A\&AS 27, 1

Smith K.C., 1995, A\&A 297, 237

Smith K.C., Dworetsky M.M., 1993, A\&A 274, 335

Sugar J., Musgrove A., 1993, J. Phys. Chem. Ref. Data 22, 1238

Takada-Hidai M., Sadakane K., Jugaku J., 1986, ApJ 304, 425

Van Regemorter H., Hoang Binh Dy, Prud'homme M., 1979, J. Phys. B 12, 1073 